\title{
Low expression of ferritinophagy-related NCOA4 gene in relation to unfavorable outcome and defective immune cells infiltration in clear cell renal carcinoma
}

\author{
Yanhua Mou ${ }^{1,2,3}$, Jinchun Wu' ${ }^{1}$, Yao Zhang ${ }^{4}$, Omar Abdihamid ${ }^{1}$, Chaojun Duan ${ }^{3,5}$ and Bin Li ${ }^{1,6^{*}}$
}

\begin{abstract}
Background: Clear cell renal cell carcinoma is susceptible to ferroptosis, and immunotherapy is recently recommended as a priority for the initial treatment of metastatic clear cell renal carcinoma. Increased ferroptosis and immune activation can synergistically reinforce each other in killing cancer cells. NCOA4 depletion can eliminate iron accumulation and thus weaken ferroptosis. Here, we aim to identify and validate the association between NCOA4 expression, clinicopathologic characteristics, and overall survival in ccRCC by using The Cancer Genome Atlas and Gene Expression Omnibus databases. We further analyze the interacted proteins of NCOA4 and infiltrated immune cells via TIMER and GEPIA databases.
\end{abstract}

Methods: NCOA4 expression in clear cell renal carcinoma (ccRCC) tissues and normal adjacent tissues in The Cancer Genome Atlas (TCGA) data were primarily screened, and further validated in another independent cohort from the gene expression omnibus (GEO) database and human protein atlas. The relationships of NCOA4 expression and clinicopathologic parameters and overall survival (OS) were assessed using multivariate methods and Kaplan-Meier survival curves. And the proteins network with which NCOA4 interacted were also built using the online STRING website. Meanwhile, we use TIMER and GEPIA databases to investigate the relationships between NCOA4 expression and infiltrated immune cells and their corresponding gene marker sets.

Results: Contrast to normal tissue, NCOA4 expression was lower in cCRCC tumor tissue $(p<0.05)$. Lower NCOA4 expression was closely associated with high-grade malignancy and advanced TNM stage. Univariate and multivariate analysis indicated the overall survival of ccRCC cases with low NCOA4 level is shorter than those of patients with high NCOA4 expression $(p<0.05)$. FTL and FTH1 were the important proteins interacting with NCOA4. ccRCC with NCOA4 deficiency presented the paucity of infiltrated immune cells and their matching marker sets, including CD8+ T cells.

Conclusion: Deficient NCOA4 expression was related to disease progression and poor prognosis, as well as impaired infiltration of immune cells in ccRCC.

Keywords: Ferritinophagy, NCOA4, Clear cell renal carcinoma, Immune cells, Ferroptosis

\footnotetext{
* Correspondence: bincsuxy@csu.edu.cn

'Department of Oncology, Xiangya Hospital, Central South University,

Changsha, Hunan 410008, P.R. China

${ }^{6}$ National Clinical Research Center for Geriatric Disorders, Xiangya Hospital,

Central South University, Changsha, Hunan 410008, P.R. China

Full list of author information is available at the end of the article
}

(c) The Author(s). 2021 Open Access This article is licensed under a Creative Commons Attribution 4.0 International License, which permits use, sharing, adaptation, distribution and reproduction in any medium or format, as long as you give appropriate credit to the original author(s) and the source, provide a link to the Creative Commons licence, and indicate if changes were made. The images or other third party material in this article are included in the article's Creative Commons licence, unless indicated otherwise in a credit line to the material. If material is not included in the article's Creative Commons licence and your intended use is not permitted by statutory regulation or exceeds the permitted use, you will need to obtain permission directly from the copyright holder. To view a copy of this licence, visit http://creativecommons.org/licenses/by/4.0/ The Creative Commons Public Domain Dedication waiver (http://creativecommons.org/publicdomain/zero/1.0/) applies to the data made available in this article, unless otherwise stated in a credit line to the data. 


\section{Background}

Over the past decades, the global incidence of renal cell carcinoma (RCC) is increasing which ranks first mortality among annual urologic cancers [1]. RCC is a heterogeneous cancer, most of them is clear cell RCC (ccRCC) which nearly accounts for $75-80 \%$ of RCC [2]. Surgery is the mainstay of ccRCC, which still present a high recurrence rate of $40 \%$ after radical surgery and have high mortality rate once it metastasizes to distant organs $[1,2]$.

Targeted therapies are currently one of the standard treatments for ccRCC, but almost all patients will ultimately develop disease deterioration because drug-induced cell apoptosis or autophage was escaped by ccRCC cells [3]. Therefore, as a novel cell death, ferroptosisinduction is becoming an alternative choice for the therapeutic strategy of ccRCC [4-7].

NCOA4, also named as androgen receptor-associated protein 70 (ARA70), was originally described as a coactivator of multiple nuclear hormone receptors. It was closely related to tumorigenesis and progression of ovarian cancer, prostate cancer, breast cancer and pancreatic cancer [8]. Recent studies unravel that NCOA4 is an autophagosomes component that participates in the process of ferritinophagy $[8,9]$. Surface arginine in ferritin heavy chain 1 (FTH1) can specifically bind the C-terminal element of NCOA4 and fused with a lysosome via nascent autophagosomes, thus facilitating ferroptotic cell death [10]. NCOA4 was gradually considered as a key molecule promoting ferroptosis in various cancer cells and mounting studies displayed that NCOA4 depletion can inhibit ferroptosis by eliminating the accumulation of intracellular free iron, glutathione production and reactive oxygen species (ROS) [11].

Immunotherapy is coming into clinical practice in the treatment of ccRCC due to FDA approval and NCCN recommendation [12]. Recently, two independent studies simultaneously reported the same finding that ferroptosis provoked by $\mathrm{T}$ cells in cancer cells is an important anti-tumor method of PD-1/PD-L1 antibody and weak effect of PD-L1 antibody was observed in the ferroptosisinsensitive tumor cells $[13,14]$. Increased ferroptosis can also advance the anti-tumor effect of immunotherapy which pinpointed the positive feedback between ferroptosis and immunotherapy, cooperatively killing cancer cells [13].

In our study, we downloaded and analyzed the relationship of NCOA4 expression with clinical information and overall survival in ccRCC patients utilizing various databases of TCGA, GEO and human protein atlas. And then we used TIMER and GEPIA databases to investigate the relationships between NCOA4 expression and infiltrated immune cells and their corresponding gene marker sets. Also, the NCOA4-interacted protein network was explored utilizing the STRING website. The results showed that low NCOA4 level acted as an indicator for poor prognosis and was associated with deficient infiltration of immune cells in ccRCC. Thus, it is plausible that NCOA4 defect reduce ferroptosis and thus possibly debilitate antitumor immune effects in ccRCC. Targeting NCOA4 may be a promising therapeutic strategy for ferroptosis-induction or/and with the combination of immunotherapy in ccRCC.

\section{Methods}

\section{Data source}

The Cancer Genome Atlas (TCGA) (https://genomecancer.ucsc.edu/), a free data portal of largescale cancer genome project, provides clinic and pathological information of 33 types of cancer for scholars and researchers. The data of ccRCC patients with the expression of RNA-Seq and matching clinical pathologic information were obtained by the TCGA tools cancer browser. The database is publicly open-access and available and therefore there was no need to get approval from the local ethics committee.

\section{The GEO database and the human protein atlas}

As one of the biggest collection of gene chips in the world, the GEO database is a comprehensive gene expression library in the National Center of Biotechnology Information (NCBI) (https://www.ncbi.nlm.nih.gov/geo/). The Human Protein Atlas offers a broad amount of proteomic and transcriptome information of distinct human samples, which consists of cell, tissue and pathology Atlas. To date, the online database provides cell-specific location information for 44 various normal tissues and 20 of the most typical categories of cancer. Moreover, protein immunohistochemistry in normal human tissues and tumor tissues can also be obtained from this online website.

\section{Survival and statistical analysis}

According to the median expression of NCOA4 gene, patients in test and validation set were split into two groups of high NCOA4 expression and low NCOA4 expression respectively. To investigate whether NCOA4 expression level affects the clinical outcomes of ccRCC patients, we constructed a prognostic classifier using Kaplan-Meier (KM) survival curves to compare the survival disparities.

\section{Univariate and multivariate logistic regression analysis}

To further determine the effect of NCOA4 expression in ccRCC patients, we use univariate Cox regression analysis for calculating the association between the expression level of NCOA4 and patient's OS in two cohorts. Afterwards, a multivariate analysis was used to assess if the NCOA4 is an independent prognostic factor for ccRCC patient survival. The NCOA4 has statistical significance in Cox regression analysis when $p$ value is less than 0.05 . 
Protein-protein interaction comprehensive analysis Another online tool we used was the Search Tool for the Retrieval of Interacting Genes/Proteins (STRING) website (https://string-db.org/). The website hosts a big collection of integrated and consolidated protein-protein interaction data. After importing the NCOA4 into the online tool STRING, we obtained the protein-protein interaction (PPI) network information. The confidence score $>0.7$ was considered significant.

\section{TIMER database analysis}

The Tumor Immune Estimation Resource (TIMER) is a public website which covers 32 cancer types and encompasses 10,897 samples from TCGA database, aiming to assess the abundance of immune inner infiltrates (http:// cistrome.org/TIMER/). The correlation of NCOA4 expression with the abundance of six types of infiltrating immune cells (CD8+ T cells, CD4+ T cells, B cells, dendritic cells macrophages, and neutrophils) in ccRCC patients was evaluated via TIMER database. The relationship between the expression of the NCOA4 gene and the tumor purity was also displayed.

\section{Gene correlation analysis}

The Gene Expression Profiling Interactive Analysis (GEPI A) (http://gepia.cancer-pku.cn/index.html) is an online database that consists of 9736 tumors and 8587 normal samples from TCGA and GTEx data. It focuses on the analyses of the expression of RNA sequencing. Gene Classes and Isoform Classes exhibit the types of 60,498 genes and 198,619 isoforms. In the GEPIA database, the relation of NCOA4 expression with multiple markers for immune cells was investigated. The $\mathrm{x}$-axis was presented with the level of NCOA4 expression, and the $y$-axis was plotted with other interest genes. In addition, we used TIMER data to validate the genes which were of significant correlation with NCOA expression in the GEPIA web.

\section{Results}

\section{Patient characteristics}

In total, the RNA-sequencing data and detailed clinical prognostic information resources of 533 ccRCC samples and 72 normal tissue samples from the TCGA database were incorporated into our research. All patients were randomly grouped into test set $(n=355)$ and validation set $(n=178)$. We summarized the clinical information including age at diagnosis, gender, laterality, histologic grade, pathologic stage $(\mathrm{T}, \mathrm{N}$ or $\mathrm{M})$, OS time and survival outcomes in Table 1.

\section{Lower NCOA4 expression in tumor samples than that in} normal tissues

The mRNA expression level of NCOA4 was analyzed in various cancer types. The gene expression level of NCOA4 was significantly lower in tumor samples in comparison to normal tissues of ccRCC in TCGA database $(p=7.337 \mathrm{e}-2)$ (Fig. 1), which was also validated in GEO database $(p=$ 4.696e-05, 0.018) (Fig. 2). Correspondingly, the expression of NCOA4 protein is downregulated in ccRCC tissue as compared to normal tissue in comparison to that in normal tissue in the Human Protein Atlas. In the analysis of the correlation of NCOA4 expression and clinicopathologic parameters in ccRCC patients, the results show that no significant difference between NCOA4 mRNA levels and age $(p=0.879)$, gender $(p=0.651)$ and pathologic $\mathrm{N}$ stage $(p=0.113)$. But lower NCOA4 expression level was observed in higher $\mathrm{T}$ stage and $\mathrm{M}$ stage and tumor stage $(p=1.917 \mathrm{e}-05,7.948 \mathrm{e}-04,7.978 \mathrm{e}-06)$, as well as in higher grade and ccRCC classification ( $p=2.428 \mathrm{e}-04,2.9283 \mathrm{e}-08)$.

\section{Lower NCOA4 mRNA expression showing shorter OS in CCRCC}

According to the KM plots, ccRCC cases with lower NCOA4 mRNA expression have shown a shorter overall survival (OS) in the test cohort $(p=1 \mathrm{e}-5)$, and was also be validated in an independent ccRCC cohort $(p=8 \mathrm{e}-4)$ (Fig. 3). In the univariate Cox model, both low NCOA4 expression and high pathologic grade and stage (TNM) were a negative predictor for OS in ccRCC patients which was confirmed in the test set and validation set. Intriguingly, in multivariate regression analysis, NCOA4 expression was independent factor correlated with OS both in the test set $(p<0.01)$ and validation set $(p=0.008)$ (Fig. 4).

\section{Constructing protein interaction networks}

The functional interaction between proteins is necessary for the molecular mechanism and metabolism of malignancy. Therefore, we used STRING tool to analyze the PPI network of NCOA4 protein to determine their interactions in the progression of ccRCC. The top 10 proteins and corresponding gene names, annotations and scores are listed in Fig. 5. These genes included: AR, RET, FTL, FTH1, CCDC6, PTCH1, PNF14, ESR1, PTCH2, CUX1. The RET fusion is becoming a potential novel target in solid tumors [15]. FTL and FTH11 are the main factors that regulate iron metabolism. Elevated FTH1 mRNA levels were correlated with worse prognosis of RCC patients [16].

\section{Correlation analysis between NCOA4 expression and infiltrating immune cells}

Tumor infiltrating lymphocytes affect the survival of patients with various cancers. Therefore, we analyzed the correlation of NCOA4 expression with six kinds of infiltrating immune cells (CD8+ T cells, CD4+ T cells, B cells, dendritic cells macrophages, and neutrophils) and tumor purity. The results displayed that the expression 
Table 1 Clinical characteristics of the ccRCC patients in test and validation sets

\begin{tabular}{|c|c|c|c|}
\hline Clinical factor & $\begin{array}{l}\text { Test set } \\
(n=355)\end{array}$ & $\begin{array}{l}\text { Validation set } \\
(n=178)\end{array}$ & $\begin{array}{l}\text { Overall } \\
(n=533)\end{array}$ \\
\hline \multicolumn{4}{|l|}{ Age } \\
\hline Mean & 60.73802817 & 60.40449438 & 60.62664165 \\
\hline Median[min, max] & $61[26,88]$ & $60[29,90]$ & $61[26,90)$ \\
\hline \multicolumn{4}{|l|}{ Gender } \\
\hline Male & $235(66.2 \%)$ & $68(38.2 \%)$ & $303(56.8 \%)$ \\
\hline Female & $120(33.8 \%)$ & $110(61.8 \%)$ & $230(43.2 \%)$ \\
\hline \multicolumn{4}{|l|}{ Overall Survival time } \\
\hline Mean & 1401.886686 & 1243.398876 & 1348.758945 \\
\hline Median[min, max] & $1230[3,4537]$ & $1131.5[2,3987]$ & $1191[2,4537]$ \\
\hline Missing & 2 & 0 & 2 \\
\hline \multicolumn{4}{|l|}{ Survival State } \\
\hline Living & $240(67.6 \%)$ & $116(65.2 \%)$ & $356(66.8 \%)$ \\
\hline Dead & 113(31.8\%) & $62(34.8 \%)$ & 175(32.8\%) \\
\hline Missing & $2(0.6 \%)$ & $0(0 \%)$ & $2(0.4 \%)$ \\
\hline \multicolumn{4}{|l|}{ Laterality } \\
\hline Left & $170(47.9 \%)$ & $81(45.5 \%)$ & $251(47.1 \%)$ \\
\hline Right & 184(51.8\%) & $97(54.5 \%)$ & $281(52.7 \%)$ \\
\hline Missing & $1(0.3 \%)$ & $0(0 \%)$ & $1(0.2 \%)$ \\
\hline \multicolumn{4}{|l|}{ Histologic grade } \\
\hline G1 & $10(2.8 \%)$ & $4(2.2 \%)$ & $14(2.6 \%)$ \\
\hline $\mathrm{G} 2$ & $152(42.8 \%)$ & $77(43.3 \%)$ & $229(43.0 \%)$ \\
\hline G3 & 139(39.2\%) & $67(37.6 \%)$ & 206(38.6\%) \\
\hline G4 & $51(14.4 \%)$ & $25(14.1 \%)$ & $76(14.3 \%)$ \\
\hline Missing & $3(0.8 \%)$ & $5(2.8 \%)$ & $8(1.5 \%)$ \\
\hline \multicolumn{4}{|l|}{ Pathologic T } \\
\hline $\mathrm{T} 1$ & $180(50.7 \%)$ & $93(52.3 \%)$ & $273(51.2 \%)$ \\
\hline $\mathrm{T} 2$ & $51(14.4 \%)$ & 18(10.1\%) & $69(12.9 \%)$ \\
\hline T3 & 118(33.2\%) & $62(34.8 \%)$ & 180(33.8\%) \\
\hline T4 & $6(1.7 \%)$ & $5(2.8 \%)$ & $11(2.1 \%)$ \\
\hline \multicolumn{4}{|l|}{ Pathologic N } \\
\hline No & 164(46.2\%) & $76(42.7 \%)$ & $240(45.0 \%)$ \\
\hline N1 & $11(3.1 \%)$ & $5(2.8 \%)$ & $16(3.0 \%)$ \\
\hline Missing & 180(50.7\%) & $97(54.5 \%)$ & $277(52.0 \%)$ \\
\hline \multicolumn{4}{|l|}{ Pathologic M } \\
\hline MO & 284(80.0\%) & 138(77.5\%) & $422(79.2 \%)$ \\
\hline M1 & $48(13.5 \%)$ & $31(17.4 \%)$ & 79(14.8\%) \\
\hline Missing & $23(6.5 \%)$ & $9(5.1 \%)$ & $32(6.0 \%)$ \\
\hline \multicolumn{4}{|l|}{ Pathologic stage } \\
\hline 1 & 175(49.3\%) & $92(51.7 \%)$ & $267(50.1 \%)$ \\
\hline ॥ & $45(12.7 \%)$ & $12(6.7 \%)$ & $57(10.7 \%)$ \\
\hline III & $83(23.4 \%)$ & $40(22.5 \%)$ & $123(23.1 \%)$ \\
\hline IV & $50(14.1 \%)$ & 34(19.1\%) & 84(15.7\%) \\
\hline Missing & $2(0.5 \%)$ & $0(0 \%)$ & $2(0.4 \%)$ \\
\hline
\end{tabular}




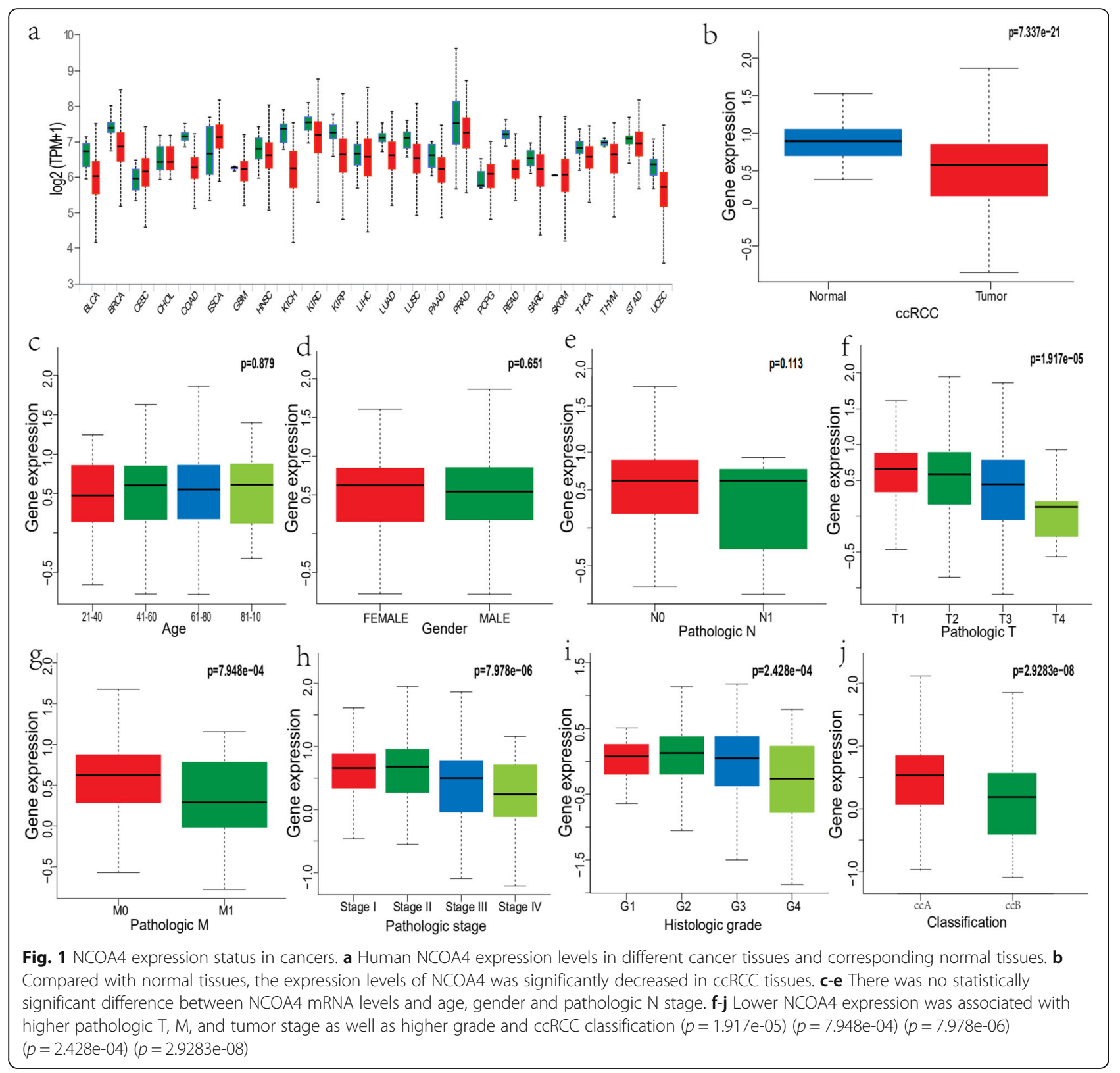

level of NCOA4 had obviously positive correlation with infiltrating levels of B cells $(r=0.304, P=2.79 \mathrm{e}-11)$, CD8 + T cells $(r=0.186, P=9.32 \mathrm{e}-05)$, macrophages $(r=$ $0.477, P=8.65 \mathrm{e}-27)$, neutrophils $(r=0.29, P=2.57 \mathrm{e}-10)$, and dendritic cells $(r=0.326, P=9.65 \mathrm{e}-13)$ in ccRCC, but no association with tumor purity and CD4+ T cells. $P<0.05$ was considered as the difference is of significance (Fig. 6).

However, there was no significant correlation between NCOA4 expression with the amount of infiltrated CD4+ $\mathrm{T}$ cells and tumor purity in ccRCC. To intensely explore the possible role of NCOA4 in the infiltration of various immune cells in ccRCC, we used the GEPIA and TIMER databases to execute the relationships between NCOA4 and several immune marker sets, which were widely accepted as corresponding symbols of different immunocytes, such as CD8+ $\mathrm{T}$ cells, $\mathrm{T}$ cells (general), $\mathrm{B}$ cells, M1/M2 macrophages, tumor-associated macrophages, neutrophils, monocytes, NK, and DCs in ccRCC (Table 2). Furthermore, various functional $\mathrm{T}$ cells including Th1, Th2, Th9, Th17, Th22, Tfh, exhausted $\mathrm{T}$ cells, and Treg, were also be examined in our study. Results showed that the levels of most immune sets marking different $\mathrm{T}$ cells, TAMs, M1/ M2 macrophages, monocytes and DCs were associated with the NCOA4 expression in ccRCC. 

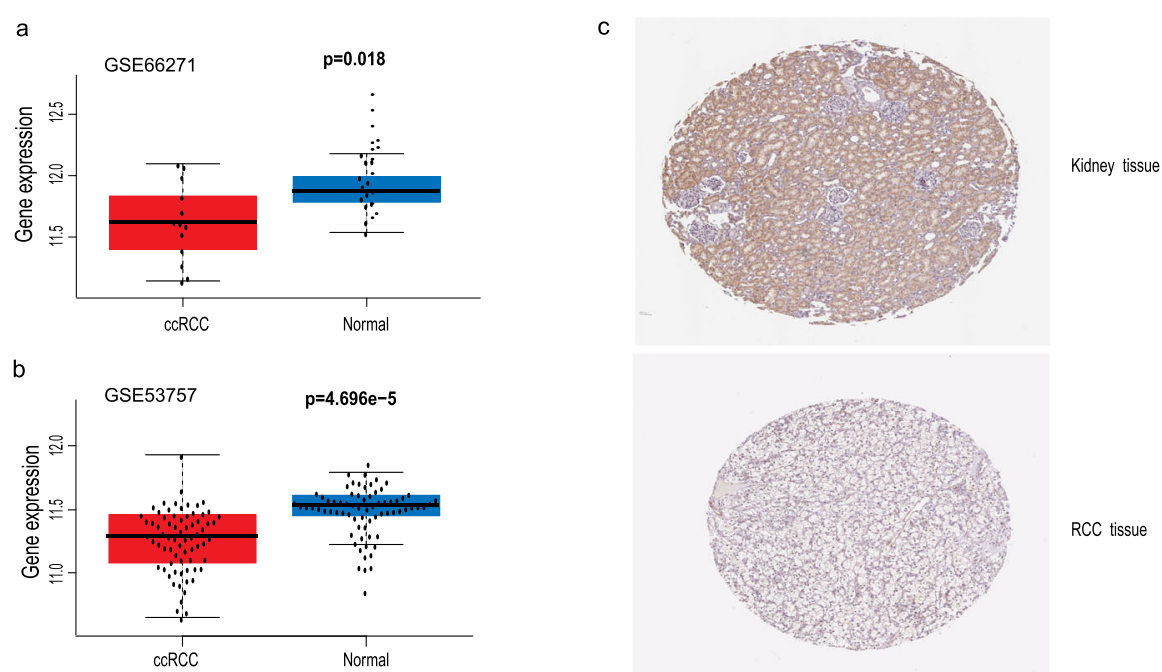

Fig. 2 Analysis of the NCOA4 gene expression in GEO datasets and the human Protein atlas. a Validation of lower NCOA4 mRNA expression in ccRCC than that in normal tissue in GSE66271 dataset. b Validation of lower NCOA4 mRNA expression in cCRCC than that in normal tissue in GSE53757 dataset. c The level of NCOA4 protein in RCC tissue was lower than that in normal tissue in the Human Protein Atlas (Antibody HPA065208, 10X)

\section{Discussion}

NCOA4 is a cargo receptor, which is specific for ferritin turnover by expediting ferritinophagy, thus it is crucial for iron homeostasis [9]. It promotes cell ferroptosis by degrading intracellular ferritin and causing iron retention, which indicates that NCOA4 is an important molecule in the process of ferroptosis in cancer [17]. NCOA4 depletion was reported to cause a cell disturbed ferroptosis process by eliminating the accumulation of intracellular free iron, glutathione and reactive oxygen species (ROS), and it was closely related to the tumorigenesis and progression of various cancers such as prostate cancer, ovarian cancer and breast cancer $[8,18]$.

ccRCC is the most common subtype of RCC [1]. More than $90 \%$ of ccRCC tumors show constitutive activation of the hypoxia-inducible factor (HIF) proteins resulting from biallelic inactivation of the tumor suppressor von
Hippl-Lindau (VHL) gene, which underline the clear-cell phenotype of ccRCC because of abnormal lipid and glycogen accumulation, also accounting for its trait of resistance to chemotherapy and radiotherapy [19-22]. Recently, growing studies unraveled that ccRCC hold an innate susceptibility to ferroptosis because of its special metabolic states derived from the hypoxia-inducible factor pathway in ccRCC $[6,7]$.

Herein, we execute the study about the role of NCOA4 expression on tumorigenesis and progression, as well as the prognosis of ccRCC on the basis of various databases including TCGA, GEO and Human Protein Atlas. Expectedly, deficient NCOA4 was associated with the tumorigenesis and progression of ccRCC. ccRCC cases with lower NCOA4 expression showed inferior prognosis in comparison to that with higher NCOA4 expression. And correspondingly, as functionally distinct
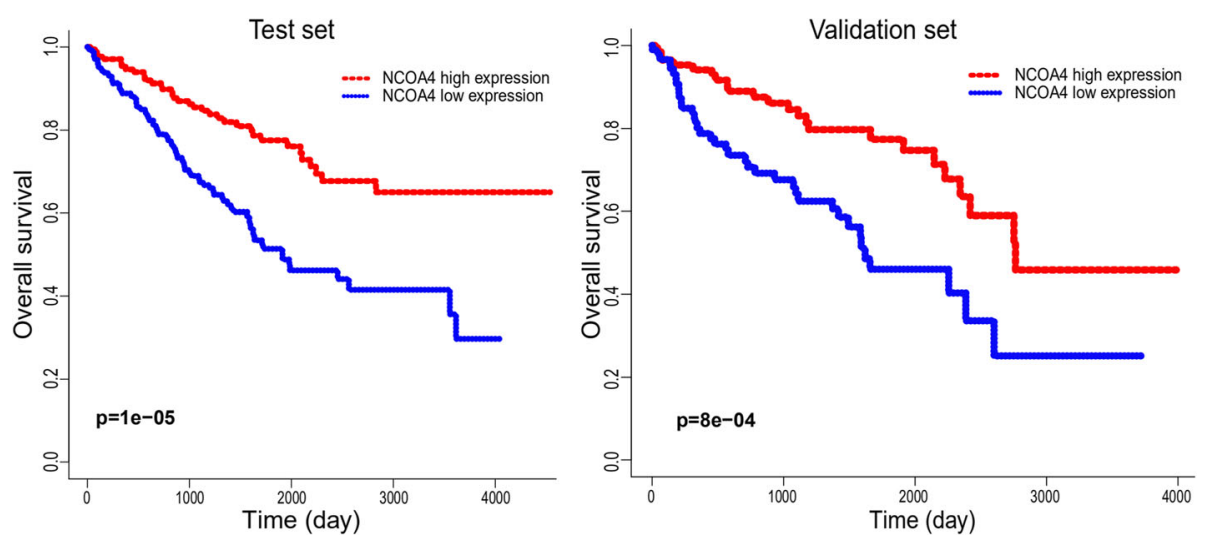

Fig. 3 The Kaplan-Meier survival curves of the ccRCC patients with high and low NCOA4 expression level 


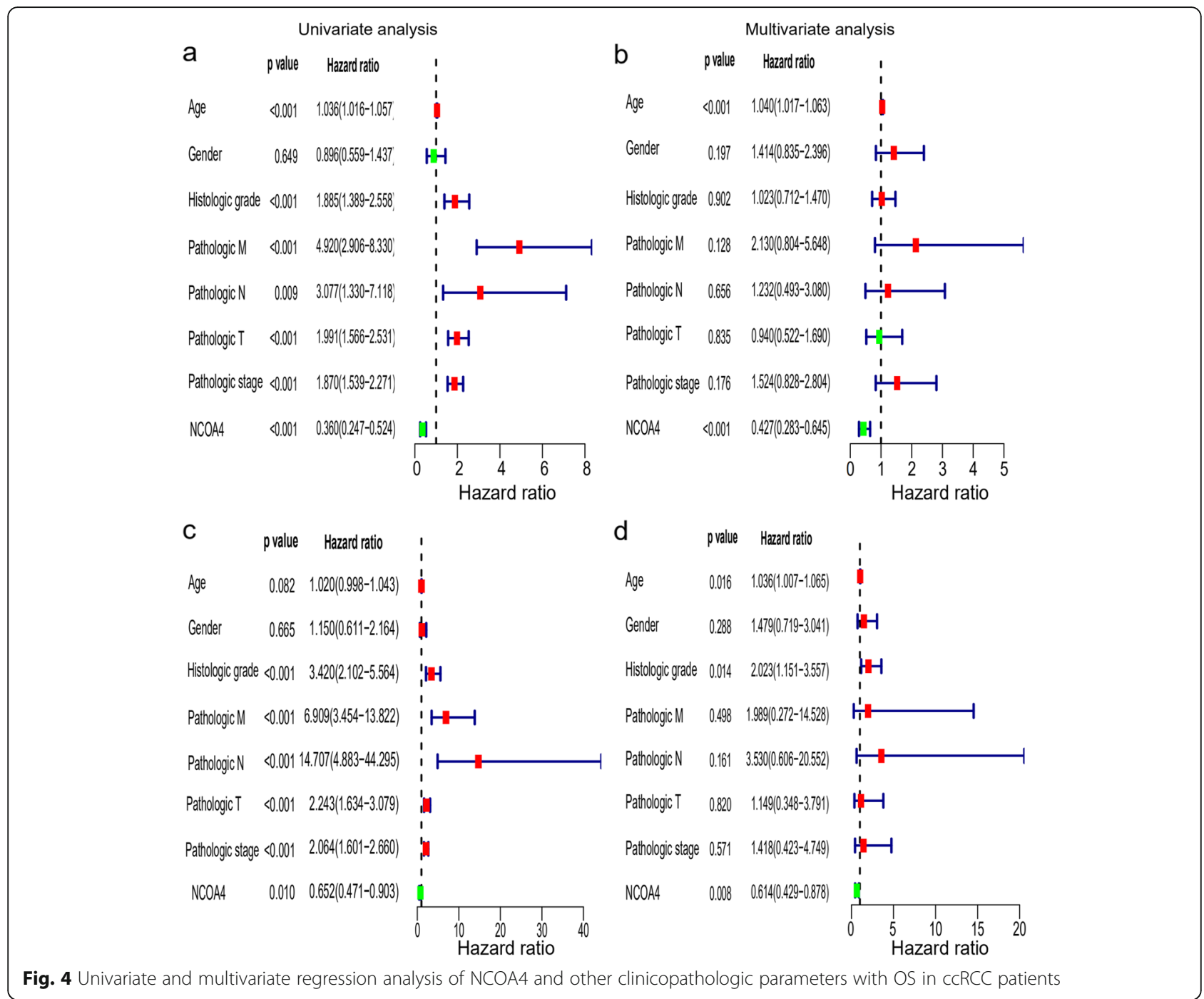

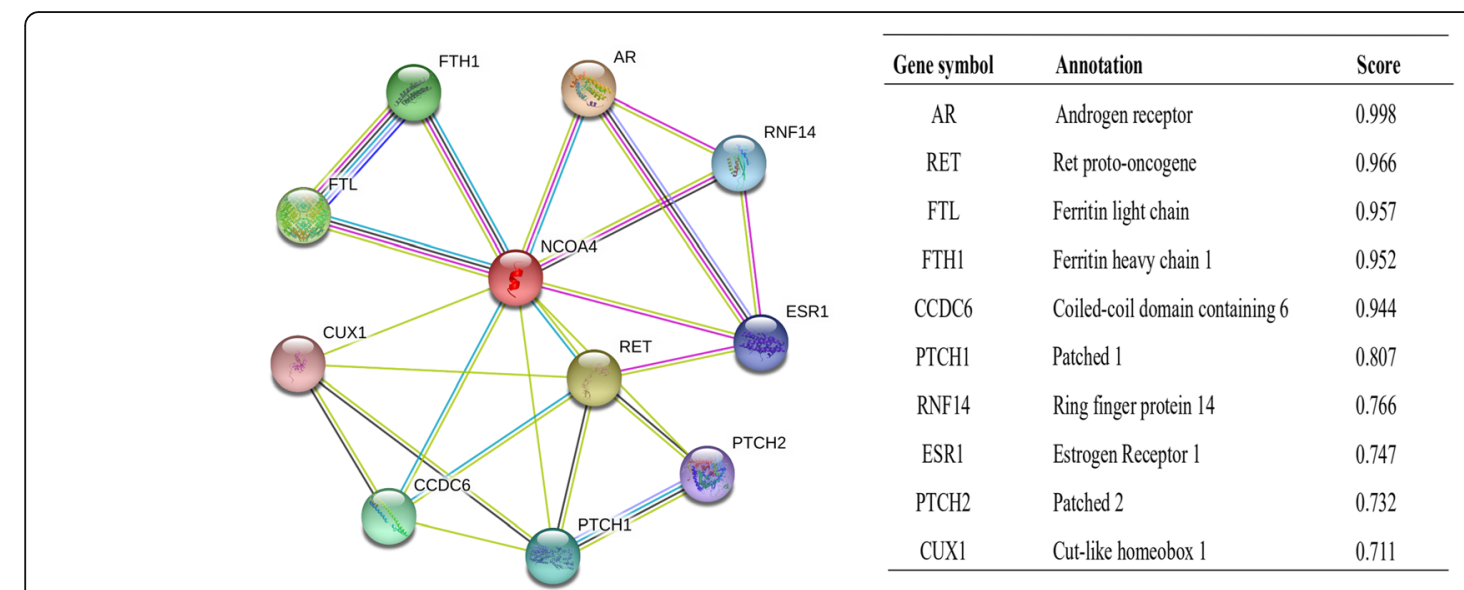

Fig. 5 NCOA4-interaction proteins in ccRCC tissue. Annotation of NCOA4-interacting proteins and their co-expression scores 


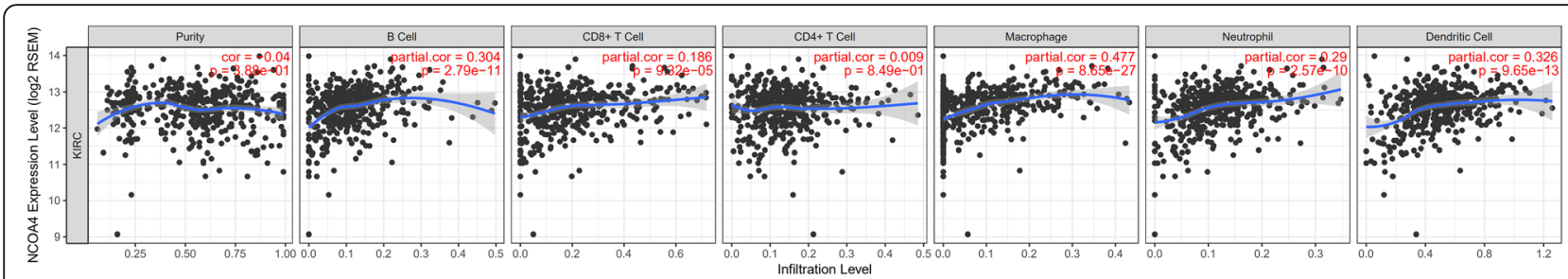

Fig. 6 Correlation of NCOA4 expression with infiltrating immune infiltration in ccRCC

compositions of ferritin, FTL and FTH1 were also identified as the important proteins which interacted with NCOA4 molecule in ccRCC based on the analysis from STING software. Interestingly, sorafenib can induce cell ferroptosis of hepatic stellate cells (HSCs) by remarkably increasing NCOA4 expression, and thus improved the survival of the patients [23, 24]. These results indicated impaired ferroptosis resulting from NCOA4 deficiency may be the underlying mechanism for impaired NCOA4 as a negative predictor of ccRCC. Indeed, targeted ferritinophgic flux (NCOA4/ferritin) either by TGF- $\beta 1$ or combined with DpdtpA has exhibited a remarkable antitumor effect $[25,26]$.

RCC has stood out as one of the most immuneinfiltrated tumors, and clinically PD-1/PD-L1 antibody has been approved in the front-line setting of metastatic ccRCC [27]. However, although with convinced efficacy, some patients were still nonreactive to PD-1 antibody [28]. Recent study suggested that tumor infiltration lymphocytes with the state of $\mathrm{T}$ cell activation are strong prognostic determinants of ccRCC [29].

Our study demonstrated that the expression level of NCOA4 has a significantly consistent correlation with the infiltration levels of $\mathrm{B}$ cell, macrophages, dendritic cells and neutrophils in ccRCC. Further analysis of infiltrated lymphocyte markers showed that the markers of M1 macrophage such as NOS2, IRF5 and PTGS2 were weakly correlated with NCOA4 expression, whereas the gene markers of M2 macrophages such as MS4A4A, MRC1 and CD163 have a moderate relationship with NCOA level, which indicates the possible regulation role of NCOA4 in the polarization of TAM. Intriguingly, we found a strong correlation between NCOA4 and Tim-3, a vital marker gene of T-regulatory (Treg) cell exhaustion. Treg cells are notoriously known as the main manipulator creating immunosuppressive tumor microenvironment, and Treg cells infiltration within tumors were related to a higher pathological stage and poor prognosis of ccRCC [30, 31].

Compellingly, our finding unraveled that NCOA4 expression was strongly correlated with CD8+ T cells infiltration and its corresponding markers CD8A in ccRCC. CD8+ T cells are well-known effector cells of cancer immunotherapy [32, 33]. Traditionally, activated cytotoxic CD8+ $\mathrm{T}$ cells eliminate tumors mostly via irritating cell death in a Fas-Fas ligand pathways or by releasing perforin-granzyme $[34,35]$. The latest two studies individually reported that $\mathrm{CD} 8+\mathrm{T}$ cells activated by immunotherapy can induce ferroptosis by specifically enhancing lipid peroxidation in tumor cells, and that increased ferroptosis contributed to the antitumor efficacy of immunotherapy, further confirming the crucial role of ferroptosis in immunotherapy [13, 14]. Intriguingly, NCOA4 deficiency can impair the IFN- $\gamma$ receptor signaling, which is a major effector of activated $\mathrm{T}$ cells for inducing ferroptosis in immunotherapy [36]. These results further implied that NCOA4 was the key molecule for bridging ferroptosis process and immunotherapy (Fig. 7).

Ferroptosis was gradually considered as a promising modality for developing effective combinational therapy strategies in the advancing era of cancer treatment [14, 37]. Due to the evolving first-line treatment choices in the patients with metastatic ccRCC, tumor biology, and tumor microenvironment should be considered upfront in predicting the optimum benefit from treatment strategies [26, 38, 39]. NCOA4 expression could be a potential novel factor for the stratification of ccRCC patients in guiding ferroptosis or/ and immunotherapy, which may be one of the main elements in a panel that reproducibly prognosticate the patients with ccRCC.

Notable limitations of our study include that lack of an already existing large quantity of tumor and normal samples analyses from the database, data heterogeneity, and platform differences. Our study shows that pathological grade and TNM stage are not independent prognostic factors for OS in patients with ccRCC, which is different from other studies [40]. This discrepancy may be due to data heterogeneity or the different grading and staging standards [41]. In addition, the group ethics information in the TCGA database is mainly limited to white and black populations, so it is hard to extrapolate these findings to other ethnicities. Prospective efforts focusing on the validation of the results drawn from the bioinformatics prediction, including proteins detection with a western blot or immunohistochemical staining and the functional analysis of NCOA4 in facilitating ferroptosis and immunotherapy in vivo and in vitro is further needed to advance the field. 
Table 2 Correlation analysis between NCOA4 and markers of immune cells in TIMER and GEPIA

\begin{tabular}{|c|c|c|c|c|c|c|c|c|c|}
\hline \multirow[t]{2}{*}{ Cell type } & \multirow[t]{2}{*}{ Gene marker } & \multicolumn{2}{|l|}{ None } & \multicolumn{2}{|l|}{ Purity } & \multicolumn{2}{|c|}{ Tum our } & \multicolumn{2}{|l|}{ Normal } \\
\hline & & Cor & $P$ & Cor & $P$ & $\mathrm{R}$ & $P$ & $\mathrm{R}$ & $P$ \\
\hline \multirow[t]{3}{*}{ B cell } & CD19 & -0.068 & 0.119 & -0.069 & 0.139 & -0.072 & 0.1 & -0.16 & 0.18 \\
\hline & CD20(KRT20) & -0.023 & 0.593 & -0.014 & 0.771 & 0.073 & 0.096 & -0.087 & 0.47 \\
\hline & CD38 & 0.304 & $* * *$ & 0.299 & $* * *$ & 0.13 & $* *$ & 0.081 & 0.5 \\
\hline \multirow[t]{2}{*}{ CD8+ T cell } & CD8A & 0.112 & $* *$ & 0.089 & 0.0567 & 0.11 & * & -0.15 & 0.22 \\
\hline & CD8B & 0.074 & 0.0897 & 0.046 & 0.329 & 0.09 & * & -0.23 & 0.053 \\
\hline \multirow[t]{3}{*}{ Tfh } & BCL6 & 0.076 & 0.0807 & 0.066 & 0.159 & 0.17 & $* * *$ & 0.13 & 0.27 \\
\hline & ICOS & Cor & $* * *$ & 0.203 & $* * *$ & 0.19 & $* * *$ & -0.059 & 0.62 \\
\hline & CXCR5 & -0.034 & 0.431 & -0.022 & 0.634 & -0.52 & $* * *$ & -0.29 & * \\
\hline \multirow[t]{7}{*}{ Th1 } & T-bet (TBX21) & 0.061 & 0 & 0.04 & 0.393 & 0.08 & 0.067 & -0.14 & 0.26 \\
\hline & STAT4 & 0.065 & 0.136 & 0.052 & 0.269 & 0.1 & * & -0.016 & 0.89 \\
\hline & IL12RB2 & 0.241 & $* * *$ & 0.216 & $* * *$ & 0.25 & $* * *$ & 0.093 & 0.44 \\
\hline & WSX1(IL27RA) & -0.069 & 0.113 & -0.1 & * & -0.034 & 0.43 & 0.072 & 0.55 \\
\hline & STAT1 & 0.484 & $* * *$ & 0.476 & $* * *$ & 0.42 & $* * *$ & 0.26 & * \\
\hline & IFN- $\gamma($ IFNG) & 0.027 & 0.531 & 0.005 & 0.917 & 0.043 & 0.33 & -0.16 & 0.17 \\
\hline & TNF-a (TNF) & 0.146 & $* * *$ & 0.154 & $* * *$ & 0.15 & $* * *$ & 0.17 & 0.15 \\
\hline \multirow[t]{4}{*}{ Th2 } & GATA3 & -0.072 & 0.955 & -0.042 & 0.364 & -0.085 & 0.053 & 0.3 & $* *$ \\
\hline & CCR3 & 0.203 & $* * *$ & 0.205 & $* * *$ & 0.092 & * & -0.033 & 0.78 \\
\hline & STAT6 & 0.377 & $* * *$ & 0.367 & $* * *$ & 0.3 & $* * *$ & 0.22 & 0.069 \\
\hline & STAT5A & 0.355 & $* * *$ & 0.344 & $* * *$ & 0.38 & $* * *$ & 0.15 & 0.2 \\
\hline \multirow[t]{3}{*}{ Th9 } & TGFBR2 & 0.62 & $* * *$ & 0.593 & $* * *$ & 0.59 & $* * *$ & -0.089 & 0.46 \\
\hline & IRF4 & 0.151 & $* * *$ & 0.151 & $* *$ & 0.04 & 0.36 & -0.12 & 0.3 \\
\hline & PU.1(SPI1) & 0.063 & 0.144 & 0.047 & 0.318 & 0.083 & 0.058 & -0.082 & 0.49 \\
\hline \multirow[t]{4}{*}{ Th17 } & STAT3 & 0.599 & $* * *$ & 0.592 & $* * *$ & 0.6 & $* * *$ & 0.33 & $* *$ \\
\hline & $\mathrm{IL}-21 \mathrm{R}$ & 0.151 & $* * *$ & 0.142 & $* *$ & 0.11 & * & -0.19 & 0.11 \\
\hline & $\mathrm{IL}-23 \mathrm{R}$ & 0.2 & $* * *$ & 0.21 & $* * *$ & 0.13 & $* *$ & 0.014 & 0.91 \\
\hline & IL-17A & -0.052 & 0.233 & -0.026 & 0.581 & -0.013 & 0.78 & 0.071 & 0.55 \\
\hline \multirow[t]{2}{*}{ Th22 } & CCR10 & -0.18 & $* * *$ & -0.185 & $* * *$ & -0.13 & $* *$ & -0.18 & 0.14 \\
\hline & AHR & 0.537 & $* * *$ & 0.534 & $* * *$ & 0.58 & $* * *$ & 0.23 & 0.05 \\
\hline \multirow[t]{3}{*}{ Treg } & FOXP3 & -0.082 & 0.0572 & -0.096 & * & -0.11 & * & -0.05 & 0.68 \\
\hline & CD25(IL2RA) & 0.312 & $* * *$ & 0.293 & $* * *$ & 0.1 & * & -0.025 & 0.83 \\
\hline & CCR8 & 0.211 & $* * *$ & 0.207 & $* * *$ & 0.17 & $* * *$ & -0.099 & 0.41 \\
\hline \multirow[t]{4}{*}{$\mathrm{T}$ cell exhaustion } & PD-1 (PDCD1) & -0.073 & 0.0939 & -0.092 & * & -0.011 & 0.8 & -0.3 & * \\
\hline & CTLA4 & -0.002 & 0.962 & -0.005 & 0.911 & 0.017 & 0.69 & -0.038 & 0.75 \\
\hline & LAG3 & -0.074 & 0.0882 & -0.088 & 0.0578 & -0.022 & 0.61 & 0.14 & 0.23 \\
\hline & TIM-3 (HAVCR2) & 0.366 & $* * *$ & 0.337 & $* * *$ & 0.31 & $* * *$ & -0.055 & 0.65 \\
\hline \multirow[t]{2}{*}{ Macrophage } & CD68 & 0.335 & $* * *$ & 0.293 & $* * *$ & 0.4 & $* * *$ & -0.049 & 0.68 \\
\hline & CD11b (ITGAM) & 0.391 & $* * *$ & 0.383 & $* * *$ & 0.15 & $* * *$ & 0.018 & 0.88 \\
\hline \multirow[t]{3}{*}{ M1 } & INOS (NOS2) & 0.349 & $* * *$ & 0.324 & $* * *$ & 0.13 & $* *$ & 0.0074 & 0.95 \\
\hline & IRF5 & 0.134 & $* *$ & 0.139 & $* *$ & 0.14 & $* *$ & 0.26 & * \\
\hline & COX2(PTGS2) & 0.114 & $* *$ & 0.143 & $* *$ & -0.019 & 0.66 & 0.26 & * \\
\hline \multirow[t]{3}{*}{ M2 } & CD16 & 0.531 & $* * *$ & 0.514 & $* * *$ & 0.26 & $* * *$ & -0.029 & 0.81 \\
\hline & ARG1 & 0.069 & 0.111 & 0.046 & 0.32 & -0.019 & 0.67 & -0.21 & 0.079 \\
\hline & MRC1 & 0.583 & $* * *$ & 0.567 & $* * *$ & 0.51 & $* * *$ & 0.13 & 0.28 \\
\hline
\end{tabular}


Table 2 Correlation analysis between NCOA4 and markers of immune cells in TIMER and GEPIA (Continued)

\begin{tabular}{|c|c|c|c|c|c|c|c|c|c|}
\hline \multirow[t]{2}{*}{ Cell type } & \multirow[t]{2}{*}{ Gene marker } & \multicolumn{2}{|l|}{ None } & \multicolumn{2}{|l|}{ Purity } & \multicolumn{2}{|c|}{ Tum our } & \multicolumn{2}{|l|}{ Normal } \\
\hline & & Cor & $P$ & Cor & $P$ & $\mathbf{R}$ & $P$ & $\mathbf{R}$ & $P$ \\
\hline & MS4A4A & 0.461 & 0.0528 & 0.453 & $* * *$ & 0.45 & $* * *$ & 0.024 & 0.84 \\
\hline \multirow[t]{4}{*}{ TAM } & CCL2 & 0.084 & $* * *$ & 0.114 & * & 0.075 & $* * *$ & 0.033 & 0.78 \\
\hline & CD80 & 0.284 & $* * *$ & 0.3 & $* * *$ & 0.2 & 0.087 & 0.035 & 0.77 \\
\hline & CD86 & 0.389 & $* * *$ & 0.392 & $* * *$ & 0.44 & $* * *$ & 0.065 & 0.59 \\
\hline & CCR5 & 0.301 & $* * *$ & 0.3 & $* * *$ & 0.35 & $* * *$ & 0.16 & 0.17 \\
\hline \multirow[t]{3}{*}{ Monocyte } & CD14 & 0.154 & $* * *$ & 0.132 & $* *$ & 0.16 & $* * *$ & 0.032 & 0.79 \\
\hline & CD16(FCGR3B) & 0.355 & $* * *$ & 0.334 & $* * *$ & 0.2 & $* * *$ & 0.16 & 0.18 \\
\hline & CD115 (CSF1R) & 0.387 & $* *$ & 0.376 & $* * *$ & 0.36 & $* * *$ & 0.039 & 0.74 \\
\hline \multirow[t]{3}{*}{ Neutrophil } & CD66b (CEACAM8) & 0.122 & $* * *$ & 0.12 & * & 0.078 & $* * *$ & 0.29 & * \\
\hline & CD15(FUT4) & 0.498 & $* * *$ & 0.491 & $* * *$ & 0.46 & 0.075 & 0.024 & 0.84 \\
\hline & CD11b (ITGAM) & 0.391 & 0.0673 & 0.383 & $* * *$ & 0.15 & $* * *$ & 0.018 & 0.88 \\
\hline \multirow[t]{3}{*}{ Natural killer cell } & XCL1 & -0.079 & $* * *$ & -0.086 & 0.0639 & -0.039 & $* * *$ & -0.08 & 0.5 \\
\hline & CD7 & -0.226 & 0.107 & -0.271 & $* * *$ & -0.067 & 0.37 & -0.26 & * \\
\hline & KIR3DL1 & 0.07 & $* * *$ & 0.041 & 0.382 & 0.085 & 0.13 & -0.17 & 0.15 \\
\hline \multirow[t]{3}{*}{ Dendritic cell } & CD1C(BDCA-1) & 0.307 & $* * *$ & 0.29 & $* * *$ & 0.24 & 0.053 & -0.026 & 0.83 \\
\hline & CD141(THBD) & 0.26 & * & 0.243 & $* * *$ & 0.29 & $* * *$ & -0.15 & 0.22 \\
\hline & CD11C (ITGAX) & 0.1 & $* * *$ & 0.115 & * & 0.034 & $* * *$ & -0.0063 & 0.96 \\
\hline
\end{tabular}

Tfh Follicular helper T cell, Th T helper cell, Treg Regulatory T cell, TAM Tumor-associated macrophage. None, Correlation without adjustment. Purity, Correlation adjusted by purity. Cor, $\mathrm{R}$ value of Spearman's correlation. ${ }^{*} P<0.05 ;{ }^{* *} P<0.01 ;{ }^{* * *} P<0.001$

\section{Conclusion}

Taken together, ferroptosis induction and immunotherapy have been the major breakthroughs in ccRCC therapy. With the new progress in understanding the treatment biology and underlying resistance mechanism of tyrosine kinase inhibitors (TKIs), ferroptosis-based combination therapy attracts more attentions from researches for taking advantage of possible synergy. Our preliminary finding displayed that low expression of ferritinophagy-related NCOA4 gene was correlated with

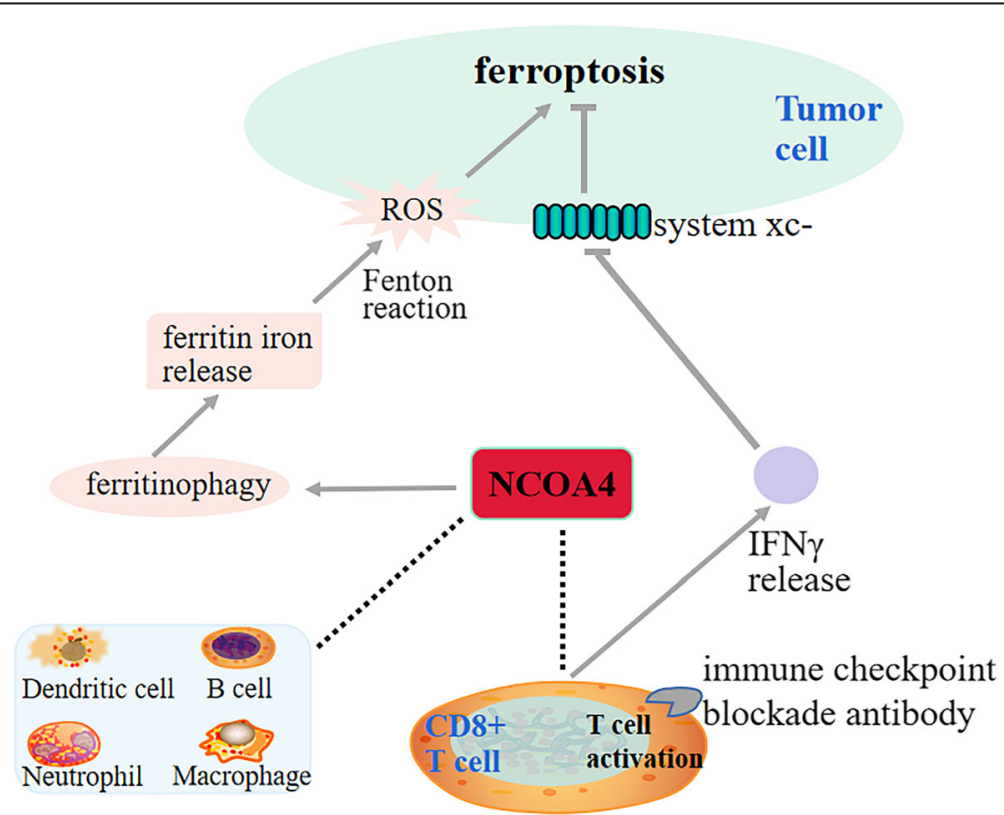

Fig. 7 Overview of the relationship between NCOA4 and ferroptosis and immune checkpoint blockade 
decreased immune cells infiltration and impaired IFN- $\gamma$ receptor signaling in ccRCC. So NCOA4 hold the expectation as a novel marker for identifying potentially eligible patients for the ferroptosis-induction treatment or its combination with immunotherapy.

\begin{abstract}
Abbreviations
ARA70: Androgen receptor-associated protein 70; ccRCC: Clear cell renal cell carcinoma; FTH1: Ferritin heavy chain 1; GEO: Gene Expression Omnibus; HIF: Hypoxia inducible factor; HSCs: Hepatic stellate cells; GEPIA: Gene Expression Profiling Interactive Analysis; NCBI: National Center of Biotechnology Information; OS: Overall survival; PPI: Protein-protein interaction; RCC: Renal cell carcinoma; ROS: Reactive oxygen species; TCGA: The Cancer Genome Atlas; TIMER: The Tumor Immune Estimation Resource; VHL: Von Hippl-Lindau
\end{abstract}

\section{Acknowledgements}

Not applicable.

\section{Authors' contributions}

$B L$ organized the article writing and critically modified the manuscript. CJD modified the manuscript. YHM drafted the manuscript and were responsible for the acquisition of data; YZ participated in the data analysis; JCW contributed to the literature search. OA check and correct language expression. All authors read and approved the manuscript and agree to be accountable for all aspects of the research in ensuring that the accuracy or integrity of any part of the work are appropriately investigated and resolved.

\section{Funding}

This work was supported by grants from the National Natural Science Foundation of China $(81200366,81572281,81702278,81974367)$ and Province Natural Science Foundation of Hunan (No.14JJ6004) and the Key Subject Education Department of Hunan ([2012]594) and Scientific Research Project of Hunan Provincial Department of Education (No. 18 K001). The funding bodies played no role in the design of the study and collection, analysis and interpretation of data and in writing the manuscript.

\section{Availability of data and materials}

The data underlying this study are freely available from TCGA data portal (https://portal.gdc.cancer.gov/projects/TCGA-KIRC) and the GSE66271 and GSE53575 dataset (http://www.ncbi.nlm.nih.gov/geo/). The authors did not have special access privileges.

\section{Ethics approval and consent to participate}

Not applicable.

\section{Consent for publication}

Not applicable.

\section{Competing interests}

The authors declare that they have no competing interests.

\footnotetext{
Author details

'Department of Oncology, Xiangya Hospital, Central South University, Changsha, Hunan 410008, P.R. China. ${ }^{2}$ Department of Oncology, Hubei Cancer Hospital, Affiliated Hubei Cancer Hospital of Huazhong University of Science and Technology, Wuhan 430079, P.R. China. Institute of Medical Sciences, Key Laboratory of Cancer Proteomics of Chinese Ministry of Health, Xiangya Hospital, Central South University, Changsha, Hunan 410008, P.R. China. ${ }^{4}$ State Key Laboratory of Reproductive Medicine and Department of Urology, The First Affiliated Hospital of Nanjing Medical University, Nanjing, Jiangsu 210029, P.R. China. ${ }^{5}$ Department of Thoracic Surgery, Xiangya Hospital, Central South University, Changsha, Hunan 410008, P.R. China. ${ }^{6}$ National Clinical Research Center for Geriatric Disorders, Xiangya Hospital, Central South University, Changsha, Hunan 410008, P.R. China.
}

Received: 29 July 2020 Accepted: 11 December 2020

Published online: 05 January 2021

\section{References}

1. Capitanio U, Bensalah K, Bex A, et al. Epidemiology of renal cell carcinoma. Eur Urol. 2019;75(1):74-84. https://doi.org/10.1016/j.eururo.2018.08.036.

2. Nabi S, Kessler ER, Bernard B, et al. Renal cell carcinoma: a review of biology and pathophysiology. F1000Res. 2018;7:307. https://doi.org/10.12688/ f1000research.13179.1.

3. Sanchez-Gastaldo A, Kempf E, Gonzalez Del Alba A, et al. Systemic treatment of renal cell cancer: a comprehensive review. Cancer Treat Rev. 2017;60:77-89. https://doi.org/10.1016/j.ctrv.2017.08.010.

4. Atkins MB, Tannir NM. Current and emerging therapies for first-line treatment of metastatic clear cell renal cell carcinoma. Cancer Treat Rev. 2018;70:127-37. https://doi.org/10.1016/.ctrv.2018.07.009.

5. Wettersten HI, Aboud OA, Lara PN, et al. Metabolic reprogramming in clear cell renal cell carcinoma. Nat Rev Nephrol. 2017;13(7):410-9. https://doi.org/ 10.1038/nrneph.2017.59.

6. Miess H, Dankworth B, Gouw AM, et al. The glutathione redox system is essential to prevent ferroptosis caused by impaired lipid metabolism in clear cell renal cell carcinoma. Oncogene. 2018;37(40):5435-50. https://doi. org/10.1038/s41388-018-0315-z.

7. Zou Y, Palte MJ, Deik AA, et al. A GPX4-dependent cancer cell state underlies the clear-cell morphology and confers sensitivity to ferroptosis. Nat Commun. 2019;10(1):1617. https://doi.org/10.1038/s41467-019-09277-9.

8. Santana-Codina N, Mancias JD. The Role of NCOA4-Mediated Ferritinophagy in Health and Disease. Pharmaceuticals (Basel). 2018;11(4). https://doi.org/10. 3390/ph11040114

9. Mancias JD, Wang X, Gygi SP, et al. Quantitative proteomics identifies NCOA4 as the cargo receptor mediating ferritinophagy. Nature. 2014 509(7498):105-9. https://doi.org/10.1038/nature13148.

10. Gryzik M, Srivastava A, Longhi G, et al. Expression and characterization of the ferritin binding domain of Nuclear Receptor Coactivator-4 (NCOA4). Biochim Biophys Acta Gen Subj. 2017;1861 (11 Pt A):2710-6. https://doi.org/ 10.1016/j.bbagen.2017.07.015

11. Bellelli R, Federico G, Matte A, et al. NCOA4 deficiency impairs systemic Iron homeostasis. Cell Rep. 2016;14(3):411-21. https://doi.org/10.1016/j.celrep. 2015.12.065.

12. Gao X, McDermott DF. Ipilimumab in combination with nivolumab for the treatment of renal cell carcinoma. Expert Opin Biol Ther. 2018;18(9):947-57. https://doi.org/10.1080/14712598.2018.1513485.

13. Wang W, Green M, Choi JE, et al. CD8(+) T cells regulate tumour ferroptosis during cancer immunotherapy. Nature. 2019;569(7755):270-4. https://doi. org/10.1038/s41586-019-1170-y.

14. Lang X, Green MD, Wang W, et al. Radiotherapy and immunotherapy promote Tumoral lipid oxidation and Ferroptosis via synergistic repression of SLC7A11. Cancer Discov. 2019;9(12):1673-85. https://doi.org/10.1158/ 2159-8290.CD-19-0338.

15. Kim SY, Oh SO, Kim K, et al. NCOA4-RET fusion in colorectal cancer: therapeutic challenge using patient-derived tumor cell lines. J Cancer. 2018; 9(17):3032-7. https://doi.org/10.7150/jca.26256 eCollection 2018

16. Huang $\mathrm{H}$, Qiu Y, Huang G, et al. Value of Ferritin Heavy Chain (FTH1) Expression in Diagnosis and Prognosis of Renal Cell Carcinoma. Med Sci Monitor. 2019;25:3700-15. https://doi.org/10.12659/MSM.914162.

17. Dowdle WE, Nyfeler B, Nagel J, et al. Selective VPS34 inhibitor blocks autophagy and uncovers a role for NCOA4 in ferritin degradation and iron homeostasis in vivo. Nat Cell Biol. 2014;16(11):1069-79. https://doi.org/10. 1038/ncb3053.

18. Hou $W$, Xie $Y$, Song $X$, et al. Autophagy promotes ferroptosis by degradation of ferritin. Autophagy. 2016;12(8):1425-8. https://doi.org/10 1080/15548627.2016.1187366.

19. Gossage L, Eisen T, Maher ER. VHL, the story of a tumour suppressor gene. Nat Rev Cancer. 2015:15(1):55-64. https://doi.org/10.1038/nrc3844.

20. Hsieh JJ, Le VH, Oyama T, et al. Chromosome 3p loss-orchestrated VHL, HIF, and epigenetic deregulation in clear cell renal cell carcinoma. J Clin Oncol. 2018. https://doi.org/10.1200/JCO.2018.79.2549.

21. Zhang J, Wu T, Simon J, et al. VHL substrate transcription factor ZHX2 as an oncogenic driver in clear cell renal cell carcinoma. Science. 2018:361(6399): 290-5. https://doi.org/10.1126/science.aap8411.

22. Makhov $\mathrm{P}$, Joshi $\mathrm{S}$, Ghatalia $\mathrm{P}$, et al. Resistance to systemic therapies in clear cell renal cell carcinoma: mechanisms and management strategies. 
Mol Cancer Ther. 2018;17(7):1355-64. https://doi.org/10.1158/1535-7163. MCT-17-1299.

23. Zhang $Z$, Yao $Z$, Wang $L$, et al. Activation of ferritinophagy is required for the RNA-binding protein ELAVL1/HuR to regulate ferroptosis in hepatic stellate cells. Autophagy. 2018;14(12):2083-103. https://doi.org/10.1080/ 15548627.2018 .1503146$.

24. Zhang Z, Guo M, Li Y, et al. RNA-binding protein ZFP36/TTP protects against ferroptosis by regulating autophagy signaling pathway in hepatic stellate cells. Autophagy. 2019:1-24. https://doi.org/10.1080/ 15548627.2019.1687985.

25. Sun Y, Li C, Feng J, et al. Ferritinophagic flux activation in CT26 cells contributed to EMT inhibition induced by a novel Iron Chelator, DpdtpA. Oxid Med Cell Longev. 2019;2019:8753413. https://doi.org/10.1155/2018/ 4928703 eCollection 2018.

26. Huang T, Sun Y, Li Y, et al. Growth inhibition of a novel Iron Chelator, DpdtC, against Hepatoma carcinoma cell lines partly attributed to Ferritinophagy-mediated Lysosomal ROS generation. Oxidative Med Cell Longev. 2018;2018:4928703. https://doi.org/10.1155/2018/4928703 eCollection 2018.

27. Motzer RJ, Escudier B, McDermott DF, et al. Nivolumab versus Everolimus in advanced renal-cell carcinoma. N Engl J Med. 2015;373(19):1803-13. https:// doi.org/10.1056/NEJMoa1510665.

28. Ascierto ML, McMiller TL, Berger AE, et al. The Intratumoral balance between metabolic and immunologic gene expression is associated with anti-PD-1 response in patients with renal cell carcinoma. Cancer Immunol Res. 2016;4(9):726-33. https://doi.org/10.1158/2326-6066.CIR16-0072.

29. Adotevi $\mathrm{O}$, Pere $\mathrm{H}$, Ravel $\mathrm{P}$, et al. A decrease of regulatory $\mathrm{T}$ cells correlates with overall survival after sunitinib-based antiangiogenic therapy in metastatic renal cancer patients. J Immunother. 2010;33(9):991-8. https:// doi.org/10.1097/CJl.0b013e3181f4c208.

30. Vuong L, Kotecha RR, Voss MH, et al. Tumor microenvironment dynamics in clear-cell renal cell carcinoma. Cancer Discov. 2019;9(10):1349-57. https:// doi.org/10.1158/2159-8290.CD-19-0499.

31. Sharabi A, Tsokos MG, Ding Y, et al. Regulatory T cells in the treatment of disease. Nat Rev Drug Discov. 2018;17(11):823-44. https://doi.org/10.1038/ nrd.2018.148.

32. Bradley CA. Immunotherapy: CD8(+) T cells - burn fat, get fit. Nat Rev Cancer. 2017;17(11):635. https://doi.org/10.1038/nrc.2017.94.

33. Durgeau A, Virk Y, Corgnac S, et al. Recent advances in targeting CD8 T-cell immunity for more effective Cancer immunotherapy. Front Immunol. 2018; 9:14. https://doi.org/10.3389/fimmu.2018.00014.

34. Barry M, Bleackley RC. Cytotoxic T lymphocytes: all roads lead to death. Nat Rev Immunol. 2002;2(6):401-9. https://doi.org/10.1038/nri819.

35. Golstein P, Griffiths GM. An early history of T cell-mediated cytotoxicity. Nat Rev Immunol. 2018;18(8):527-35. https://doi.org/10.1038/s41577-018-0009-3.

36. Sottile R, Federico G, Garofalo C, et al. Iron and ferritin modulate MHC class I expression and NK cell recognition. Front Immunol. 2019;10:224. https://doi. org/10.3389/fimmu.2019.00224 eCollection 2019.

37. Friedmann Angeli JP, Krysko DV, Conrad M. Ferroptosis at the crossroads of cancer-acquired drug resistance and immune evasion. Nat Rev Cancer 2019;19(7):405-14. https://doi.org/10.1038/s41568-019-0149-1.

38. Lopez-Beltran A, Henriques V, Cimadamore A, et al. The identification of immunological biomarkers in kidney cancers. Front Oncol. 2018;8:456. https://doi.org/10.3389/fonc.2018.00456.

39. Mikami S, Mizuno R, Kondo T, et al. Clinical significance of programmed death-1 and programmed death-ligand 1 expression in the tumor microenvironment of clear cell renal cell carcinoma. Cancer Sci. 2019;110(6): 1820-8. https://doi.org/10.1111/cas.14019.

40. Dagher J, Delahunt B, Rioux-Leclercq N, et al. Clear cell renal cell carcinoma: validation of World Health Organization/International Society of Urological Pathology grading. Histopathology. 2017;71(6):918-25. https://doi.org/10. 1111/his.13311.

41. Warren AY, Harrison D. WHO/ISUP classification, grading and pathological staging of renal cell carcinoma: standards and controversies. World J Urol. 2018;36(12):1913-26. https://doi.org/10.1007/s00345-018-2447-8.

\section{Publisher's Note}

Springer Nature remains neutral with regard to jurisdictional claims in published maps and institutional affiliations.

\section{Ready to submit your research? Choose BMC and benefit from:}

- fast, convenient online submission

- thorough peer review by experienced researchers in your field

- rapid publication on acceptance

- support for research data, including large and complex data types

- gold Open Access which fosters wider collaboration and increased citations

- maximum visibility for your research: over $100 \mathrm{M}$ website views per year

At BMC, research is always in progress.

Learn more biomedcentral.com/submissions 